Mexican Journal of Biotechnology 2020, 5(3):1-16

Journal homepage:www.mexjbiotechnol.com

ISSN:2448-6590

ORIGINAL RESEARCH

\title{
Pyocyanin biosynthesis by Pseudomonas aeruginosa using a biodiesel byproduct
}

\section{Biosíntesis de piocianina por Pseudomonas aeruginosa usando un subproducto del biodiesel}

Francisco Javier Bacame-Valenzuela ${ }^{1,2}$, Jesús Alberto Pérez-García ${ }^{2}$, Federico Castañeda-Zaldivar ${ }^{2}$, María Yolanda Reyes-Vidal' ${ }^{1,2 *}$

${ }^{1}$ CONACYT-CIDETEQ, Centro de Investigación y Desarrollo Tecnológico en Electroquímica, S.C. Parque Tecnológico Querétaro, Sanfandila, Pedro Escobedo, C. P. 76703, Querétaro, Mexico.

${ }^{2}$ Centro de Investigación y Desarrollo Tecnológico en Electroquímica, S. C. Parque Tecnológico Querétaro, Sanfandila, Pedro Escobedo, C. P. 76703, Querétaro, Mexico.

${ }^{*}$ Corresponding author

E-mail address: mreyes@cideteq.mx (M. Y. Reyes-Vidal)

Article history:

Received: 16 February 2020 / Received in revised form: 7 May 2020 / Accepted: / 7 June 2020 / Published online: 1 July 2020.

https://doi.org/10.29267/mxib.2020.5.3.1

\begin{abstract}
By-products of biodiesel production, such as crude glycerol, require options for their valorization, which allow obtaining high value products with application in several areas. To achieve this, the use of crude glycerol for the production of pyocyanin was analyzed using three strains of Pseudomonas aeruginosa, to increase the concentration of metabolite and compare it with that produced using lysogeny broth (LB medium). The effect was dependent on the strain used, shown positively by only two strains. The physiology of $P$. aeruginosa was analyzed using glycerol as the sole carbon source and the complete substrate for pyocyanin biosynthesis. The extracted compound was identified by UPLC-MS and characterized by electrochemical techniques. The voltamperometric response of the pyocyanin allowed analyzing its ability to participate in redox behaviour and the mechanisms on the electrode surface, which could be associated with adsorption processes. This study shows the potential of the strains used to obtain redox metabolites of high interest with application in bioelectrochemical systems. In
\end{abstract}


addition, the alternative to produce such metabolites using bioprocess by-products for energy generation, of great international use, was proposed.

Keywords: crude glycerol, Pseudomonas aeruginosa, pyocyanin.

\section{RESUMEN}

Los subproductos de la producción de biodiesel, como el glicerol crudo, requieren opciones para su valorización, que permitan obtener productos de alto valor con aplicación en diversas áreas. A partir de esto, se analizó el uso del glicerol crudo para la producción de piocianina utilizando tres cepas de Pseudomonas aeruginosa, con el objetivo de aumentar la concentración obtenida de este metabolito y compararla con la producida usando caldo de lisogenia (medio LB). El efecto fue dependiente de la cepa usada, mostrado de manera positiva solo por dos cepas. Se analizó la fisiología de $P$. aeruginosa usando al glicerol como única fuente de carbono y del sustrato completo para la biosíntesis de piocianina. El compuesto extraído fue identificado por UPLC-MS y caracterizado por técnicas electroquímicas. La respuesta voltamperométrica de piocianina permitió analizar su capacidad para asistir procesos redox y los mecanismos en la superficie del electrodo, que podrían estar asociados a procesos de adsorción. Este estudio muestra el potencial de las cepas usadas para la obtención de metabolitos redox con aplicación en sistemas bioelectroquimicos; además, se planteó la alternativa para producir tales metabolitos usando subproductos de bioprocesos para la generación de energéticos, de amplio uso internacional.

Palabras clave: glicerol crudo, piocianina, Pseudomonas aeruginosa.

\section{INTRODUCCIÓN}

Los combustibles fósiles, que van en aumento, son considerados necesarios para el desarrollo económico y la industrialización, pero su obtención y procesamiento están relacionados con los efectos negativos que originan el cambio climático (Sarma et al., 2019). Actualmente, existe gran interés en el desarrollo de energías renovables, como el biodiesel, que representan una alternativa a los combustibles fósiles (Chozhavendhan et al., 2018). Tan sólo en 2017, la Unión Europea y Estados Unidos obtuvieron 21 y 32 millones de toneladas de dicho combustible, respectivamente. En América Latina, Brasil genera una producción anual de 300,000 toneladas con una proyección del $5.7 \%$ de crecimiento anual, según la Agencia Nacional del Petróleo. Sin embargo, a pesar de las ventajas ambientales que tienen la producción y el uso de biodiesel, también se requiere una importante atención en los subproductos obtenidos durante su producción, siendo el glicerol crudo (GC) uno de los más importantes, ya que representa el $10 \%$ de su volumen de producción. En este escenario, es necesaria la búsqueda de alternativas para 
el uso del GC, ya que se considera altamente tóxico debido al contenido de impurezas. Así mismo, el aumento en la demanda de biodiesel hace que el precio del glicerol crudo disminuya (0.33 USD/kg) obligando a su disposición en vez de su utilización (Kondaveeti et al., 2019; Mangayil et al., 2019; Robert et al., 2017).

Desde el punto de vista económico y ambiental, la obtención de productos de valor agregado a partir del GC es de gran importancia, ya que representa una excelente fuente de carbono de la cual se pueden obtener diversos metabolitos de valor agregado (Mangayil et al., 2019). Bajo esta perspectiva, los productores de biodiesel pueden considerar la transformación del GC como una fuente de ingresos, además de la disposición del efluente de manera amigable con el ambiente (Uprety et al., 2017). Por ello, para la utilización del GC se han diseñado diferentes procesos, tanto químicos como biológicos, siendo de gran relevancia la bioconversión porque incorpora al GC sin un tratamiento previo. De esta manera el GC podría ser usado como sustrato en medios de cultivo de bajo costo, representando la única fuente de carbono o en co-digestión con otros residuos industriales como desechos de frutas y vegetales, la fracción orgánica de los sólidos de residuos municipales, estiércol de cerdo, mezcla de aguas residuales de rastros con desechos de molinos de granos y semillas, mezcla de estiércol y efluentes industriales orgánicos (Nartker et al., 2014).

Pseudomonas aeruginosa es una bacteria que utiliza fuentes de carbono complejas como las que se encuentran en efluentes industriales o aguas municipales, debido a su versatilidad metabólica, teniendo gran relevancia en procesos ambientales (Yong et al., 2017). Este microorganismo es productor de compuestos derivados de fenazina principalmente piocianina (Pio). Esta molécula, azul verdosa soluble en agua, presenta diversas actividades biológicas como la inhibición de los hongos fitopatógenos Colletrichum circinans MTCC 10329 y Sclerotinia sclerotiorum MTCC 8785, y es también conocida por su efecto de antivibriosis. En algunos estudios se ha demostrado su capacidad antimicrobiana contra cepas resistentes a vancomicina y meticilina como Staphylococcus aureus, Corynebacterium spp., Moraxella catarrhalis, Candida albicans, Candida tropicalis, Candida glabrata y Candida parapsilosi, entre otros (Patil et al., 2017; Xu et al., 2017). También se le conoce por su aplicación en biosensores, que permite la detección electroquímica de Pio en electrodos de referencia a microescala, para analizar cepas patógenas hospitalarias de P. aeruginosa (Webster \& Goluch, 2012). Esta utilidad depende de su capacidad como molécula redox. Esta capacidad de Pio también está asociada a su participación en los procesos de transferencia extracelular de electrones en celdas de combustible microbianas, una configuración ampliamente usada de los sistemas bioelectroquímicos para la producción de energías limpias y renovables como bioelectricidad (Oziat et al., 2017; Shen et al., 2014). Con estas aplicaciones, surge la importancia de producir Pio en concentraciones que no limiten su uso y con precios comercialmente accesibles. De acuerdo con El-fouly et al. (2015), las aplicaciones ilimitadas de Pio son posibles mediante su síntesis biológica usando medios del cultivo no convencionales como los desechos agroindustriales. En este trabajo se propone la 
biosíntesis de piocianina por tres cepas de $P$. aeruginosa usando como medio de cultivo al glicerol crudo, un subproducto de la producción de biodiesel.

\section{MATERIALES Y MÉTODOS}

\subsection{Cepa y condiciones de cultivo}

Las cepas utilizadas fueron $P$. aeruginosa NEJ01R, $P$. aeruginosa R5 y $P$. aeruginosa NEJ03R, previamente aisladas y caracterizadas por el Laboratorio de Microbiología Ambiental de CIDETEQ. Los medios de cultivo fueron caldo de lisogenia (LB, "Lysogeny Broth") el cual contenía $10 \mathrm{~g} / \mathrm{L}$ de peptona de caseína (BD Bioxon), $5 \mathrm{~g} / \mathrm{L}$ de extracto de levadura (BD Bioxon) y $0.05 \mathrm{~g} / \mathrm{L}$ de cloruro de sodio (J.T. Baker). El medio de cultivo incluyendo GC fue preparado mediante dilución de GC al $1 \%$ (GC1), sin ningún pretratamiento. Todos los medios fueron esterilizados en autoclave, e inoculados con $1 \mathrm{~mL}$ de caldo de fermentación de 24 $\mathrm{h}$ de cada cepa, con una $\mathrm{DO}_{600}$ de 0.1 . El medio inoculado fue incubado a $30^{\circ} \mathrm{C}$ y $150 \mathrm{rpm}$, por $48 \mathrm{~h}$. Todos los experimentos fueron realizados por triplicado para las tres cepas utilizadas. El medio GC1 diseñado para analizar el efecto de la adición de metanol al medio GC fue preparado con dilución al $1 \%$ de GC (previamente esterilizado) más $5 \%$ (v/v) de metanol (J.T. Baker).

\subsection{Determinación de biomasa y piocianina (Pio)}

La biomasa fue determinada por peso seco y reportada en $\mathrm{g} / \mathrm{L}$. La concentración de Pio fue determinada por extracción líquido-líquido con cloroformo 1:1. La fase orgánica fue separada y se adicionó un volumen igual de $\mathrm{HCl}(0.2 \mathrm{~N})$, se agitó vigorosamente para separar la fase acuosa. Posteriormente, se midió absorbancia $(520 \mathrm{~nm})$ de la fase acuosa, expresando la concentración de Pio en $\mu \mathrm{g} / \mathrm{mL}$, multiplicando el valor de absorbancia por el factor 17.1 (coeficiente de extinción molar de Pio), según el método reportado por El-fouly et al., 2015.

\subsection{Análisis estadístico}

Los datos obtenidos para la producción de biomasa y piocianina por las 3 cepas utilizadas para este trabajo son representados por el valor promedio \pm desviación estándar, como resultado de un triplicado de tres experimentos independientes. Se realizó un análisis estadístico ANOVA y una prueba de Duncan $(\alpha=0.5)$ para determinar aquellos valores con diferencia significativa (NCSS 8.0).

\subsection{Identificación de Pio mediante UPLC-MS}

Se utilizó un cromatógrafo de líquidos de ultraresolución (UPLC), con automuestreador refrigerado, bomba cuaternaria para solventes, horno para columna Acquity Clase-H y detector de masas (QDa) (Waters, México), compacto de un cuádruplo equipado con interfase de ionización por electrospray (ESI). Se utilizó una columna para UPLC marca Waters BEH C18 (50 mm x 2.1 mm i.d., 1.7 
$\mu \mathrm{m})$ a una temperatura de $30^{\circ} \mathrm{C}$. La fase móvil fue $90 \%$ acetonitrilo:agua (v/v) con $0.1 \%$ ácido fórmico, flujo $=0.2 \mathrm{~mL} / \mathrm{min}$ y $5 \mu \mathrm{L}$ de inyección. El detector QDa se usó en modo positivo con voltaje de cono $=10 \mathrm{~V}$. La temperatura de desolvatación fue $600{ }^{\circ} \mathrm{C}$. El espectro de masa $(\mathrm{m} / \mathrm{z})$ se consideró entre 100 y 300 con una frecuencia de muestreo de 5 puntos/segundo. Para la identificación de Pio se utilizaron los sobrenadantes de los cultivos en medio LB y GC1 de las tres cepas de estudio, los cuales fueron obtenidos por centrifugación de los medios de cultivo a $7500 \mathrm{rpm}$ por 10 minutos a $4{ }^{\circ} \mathrm{C}$. Como compuesto estándar se utilizó piocianina de Pseudomonas aeruginosa (Sigma-Aldrich, grado HPLC) pureza $\geq 98 \%$. Todas las muestras y estándares se filtraron por membranas de nylon de $0.2 \mu \mathrm{m}$. Los datos fueron procesados con el programa para cromatografía Empower3 de Waters.

\subsection{Determinación de la capacidad de Pio en procesos redox: Respuesta voltamperométrica}

Se realizó una modificación al método de extracción descrito previamente por Kurachi (1958). Se centrifugó el sobrenadante del cultivo de $P$. aeruginosa NEJ01R durante 15 minutos, $25^{\circ} \mathrm{C}$ y $7500 \mathrm{rpm}$. El sobrenadante se filtró con una membrana hidrofílica de $0.45 \mu \mathrm{m}$. Se adicionó cloroformo (J.T. Baker) al sobrenadante filtrado en relación 1:1, agitando para obtener la fracción orgánica. La fracción obtenida fue mezclada con una solución de $\mathrm{HCl} 0.2 \mathrm{~N}$ (relación 1:1). La nueva fracción removida fue ajustada a $\mathrm{pH}=7(\mathrm{NaOH}, 0.5 \mathrm{M})$, después se agregó cloroformo (J.T. Baker) $(1: 1, \mathrm{v} / \mathrm{v})$. Se recuperó la fracción orgánica y se añadió hexano (J.T. Baker) (relación 2:3). La mezcla se colocó en un cristalizador, permitiendo la evaporación del solvente. La piocianina obtenida $(0.14 \mathrm{mM})$ fue disuelta en una solución buffer de fosfatos $(0.1 \mathrm{M})$ a $\mathrm{pH} 7$ y temperatura ambiente $\left(25^{\circ} \mathrm{C}\right)$. Se realizó el estudio por voltamperometría cíclica en un potenciostato BioLogic ${ }^{\circledR}$ modelo VSP. Como electrodo de trabajo (Et) se utilizó un disco de carbón vítreo aislado (3 $\mathrm{mm}$ de diámetro), como electrodo de referencia (Eref) se usó $\mathrm{Ag} \mid \mathrm{AgCl}$ y como electrodo auxiliar (Ea) se empleó un alambre de platino. Los electrodos fueron integrados a una celda de vidrio con tapa hidrofóbica de teflón. El electrodo Et fue pulido entre cada experimento con un paño y polvo de alúmina de $0.3 \mu \mathrm{m}$. Todos los experimentos fueron purgados con $\mathrm{N}_{2}$ para minimizar la presencia de $\mathrm{O}_{2}$. Antes de cada experimento se llevó a cabo la compensación de la caída óhmica (IR) al $85 \%$ mediante espectroscopia de impedancia electroquímica (PEIS) a frecuencia alta. El valor de la frecuencia utilizada fue de $100 \mathrm{kHz}$ y se tomó el valor de la resistencia inicial Ru para el ajuste.

\section{RESULTADOS}

\subsection{Producción de biomasa y Pio}

Las tres cepas de $P$. aeruginosa utilizadas para este trabajo lograron metabolizar al glicerol crudo como fuente de carbono, ya que se obtuvo crecimiento bacteriano 
de las mismas en el medio GC1 (Tabla 1). La cepa NEJ03R mostró el mayor crecimiento en el medio de cultivo GC1, incrementado $5 \%$ la concentración de biomasa respecto a la concentración obtenida en el medio LB. Mientras que las cepas NEJ01R y R5 mostraron disminución del 25 y $45 \%$, respectivamente, de la biomasa obtenida en el medio LB (Tabla 1).

Las cepas NEJ01R y NEJ03R lograron producir 2 y 1.5 veces mayor concentración de Pio en el medio GC1, respectivamente, en comparación con la obtenida en el medio LB (Tabla 1). En el caso de la cepa R5, el medio GC1 presentó un efecto negativo ya que la Pio obtenida se redujo $35 \%$ de la Pio producida en el medio LB, representando la menor producción de Pio comparando las tres cepas utilizadas para este estudio.

Tabla 1. Producción de biomasa y Pio por 3 cepas de $P$. aeruginosa.

Table 1. Biomass and Pyo production by three strains of $P$. aeruginosa.

\begin{tabular}{llccc}
\hline \multirow{2}{*}{ Cepa } & \multicolumn{2}{c}{ GC1 } & \multicolumn{2}{c}{ LB } \\
\cline { 2 - 5 } & Biomasa $(\mathbf{g} / \mathbf{L})$ & $\begin{array}{c}\text { Pio } \\
(\mu \mathrm{g} / \mathrm{mL})\end{array}$ & Biomasa $(\mathbf{g} / \mathbf{L})$ & $\begin{array}{c}\text { Pio } \\
(\mu \mathrm{g} / \mathrm{mL})\end{array}$ \\
\hline NEJ01R & $0.595^{\mathrm{a}} \pm 0.004$ & $2.565^{\mathrm{a}} \pm 0.241$ & $0.785^{\mathrm{a}} \pm 0.083$ & $1.231^{\mathrm{a}} \pm 0.024$ \\
R5 & $0.511^{\mathrm{a}} \pm 0.080$ & $0.880^{\mathrm{b}} \pm 0.036$ & $0.937^{\mathrm{a}} \pm 0.012$ & $1.368^{\mathrm{a}} \pm 0.314$ \\
NEJ03R & $0.980^{\mathrm{b}} \pm 0.200$ & $2.573^{\mathrm{a}} \pm 0.036$ & $0.939^{\mathrm{a}} \pm 0.069$ & $1.710^{\mathrm{b}} \pm 0.024$
\end{tabular}

* Valores con letras diferentes en la misma columna tienen diferencia estadística significativa para un $\alpha=0.05$.

\subsection{Identificación de Pio mediante UPLC-MS}

Para la identificación de Pio producida por las cepas de estudio se utilizó cromatografía de alta resolución acoplada a un detector de masas (UPLC-MS) obteniendo un valor máximo masa/carga $(\mathrm{m} / \mathrm{z})[\mathrm{M}+\mathrm{H}]+=211 \mathrm{~m} / \mathrm{z}$. En la Fig. $1 \mathrm{~A}$ se muestra el cromatograma correspondiente al estándar de piocianina (SigmaAldrich), y en la Fig. 1B se puede observar el cromatograma de Pio extraída del sobrenadante de la fermentación con $P$. aeruginosa NEJ03R. De acuerdo con estos resultados el tiempo de retención de la muestra corresponde al del compuesto estándar (1.4 min). En la Fig. 2 se muestran dos señales a 212 y 213 $\mathrm{m} / \mathrm{z}$, relacionados con los iones isotópicos de Pio, similares al del estándar (Fig. $2 \mathrm{~A}$ ), confirmando que la molécula obtenida de los aislados corresponde a Pio. El análisis mediante UPLC-MS se realizó para la Pio extraída de cada una de las tres cepas utilizadas en este trabajo encontrando resultados similares. 

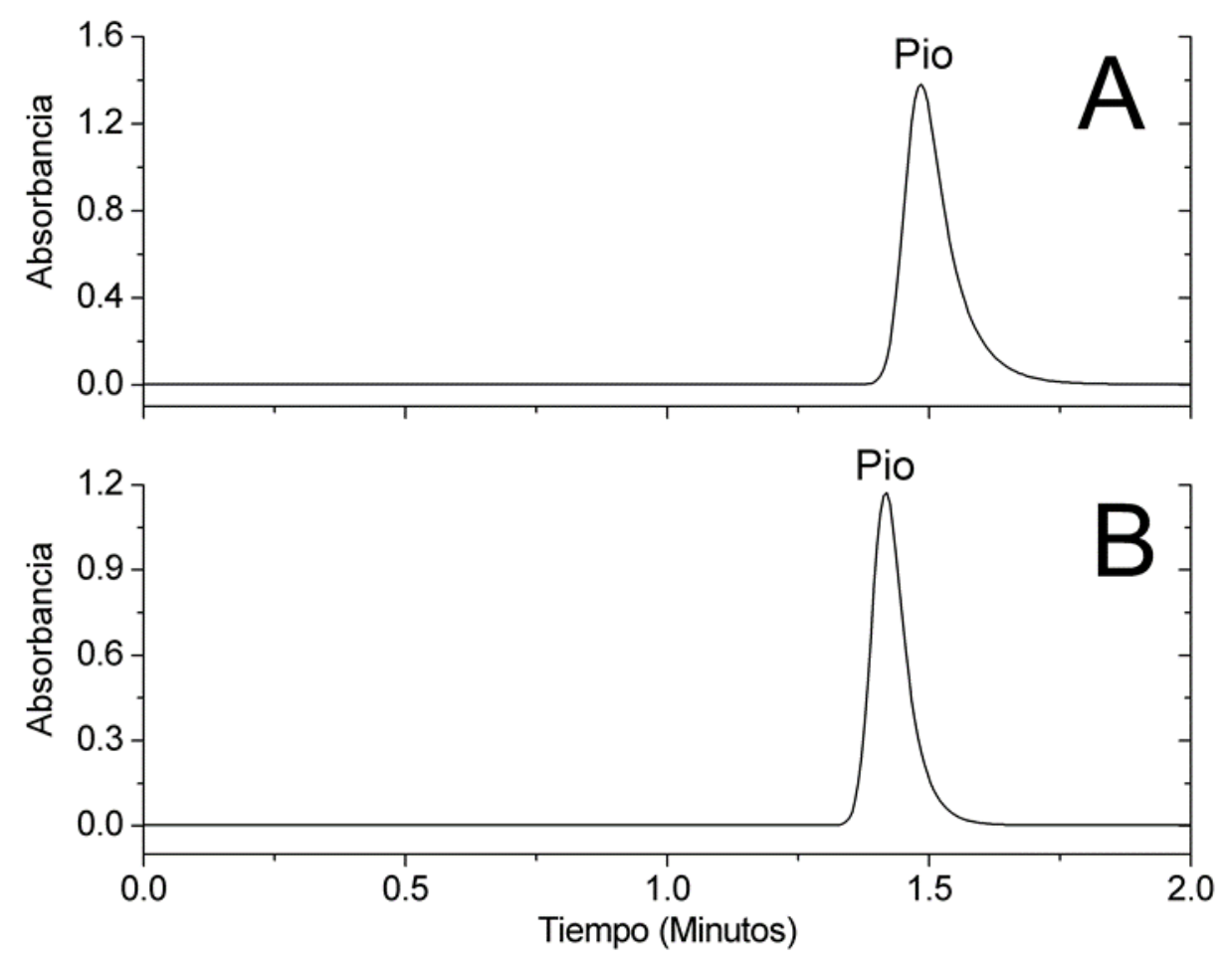

Fig. 1. (A) Cromatograma de piocianina producida por $P$. aeruginosa NEJ03R; (B) estándar de piocianina.

Fig. 1. (A) Pyocyanin chromatogram produced by P. aeruginosa NEJ03R; (B) pyocyanin standard.

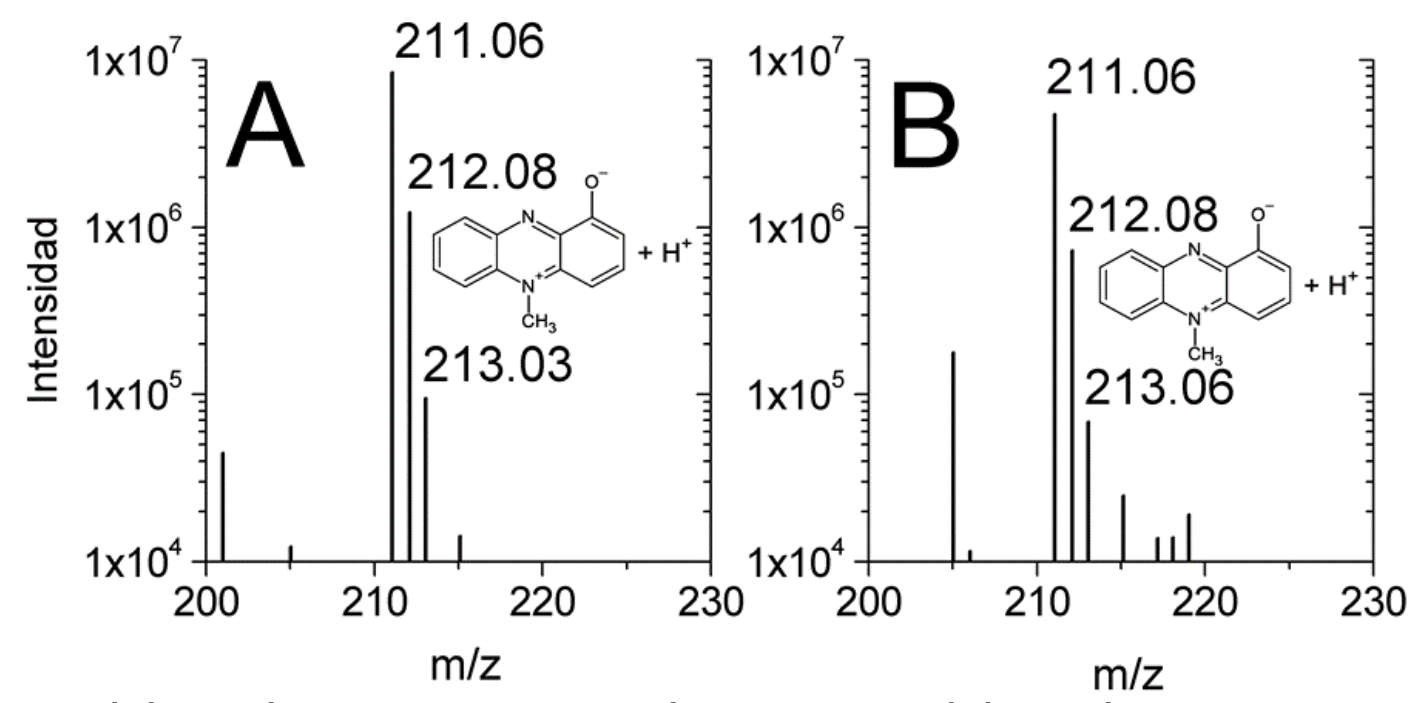

Fig. 2. (A) Patrón de masas del estándar de Pio; (B) Patrón de masas de Pio producida por $P$. aeruginosa NEJ03R.

Fig. 2. (A) Mass pattern of Pyo standard; (B) Mass pattern of Pyo produced by $P$. aeruginosa NEJ03R. 


\subsection{Determinación de la capacidad de Pio en procesos redox: Respuesta voltamperométrica}

La Fig. 3A muestra la respuesta voltamperométrica del extracto de piocianina $(0.14 \mathrm{mM})$, usando como electrolito soporte un buffer de fosfatos $100 \mathrm{mM}(\mathrm{pH} 7)$ a una velocidad de barrido $\mathrm{v}$ de $10 \mathrm{mV} / \mathrm{s}$. Durante el escaneo catódico se observó un proceso de reducción i_c con valor de E_pc $=-0.269 \mathrm{~V}$ vs $\mathrm{Ag} \mid \mathrm{AgCl}$. Al invertir el escaneo en dirección anódica se aprecia el proceso de oxidación complementario i_a con valor de E_pa $=-0.234 \mathrm{~V}$ vs $\mathrm{Ag} \mid \mathrm{AgCl}$. La respuesta originó una curva voltamperométrica característica de un sistema reversible con un valor de $\Delta \mathrm{E} \_\mathrm{p}=$ $33.8 \mathrm{mV}$, relacionado con la capacidad de la molécula para asistir procesos redox. $\mathrm{El}$ análisis del valor obtenido para $\Delta \mathrm{E} \_\mathrm{p}$ corresponde con una reacción de dos electrones de acuerdo con la relación $\Delta \mathrm{E} \_\mathrm{p}=59 \mathrm{mV} / \mathrm{n}$, donde el valor de $\Delta \mathrm{E} \_\mathrm{p}$ es la diferencia de los picos anódico y catódico $\left(\Delta E \_p=\left|E \_p a-E \_p c\right|\right)$ y el valor de $n$ corresponde al número de electrones transferidos durante la reacción electroquímica. Esta relación indica que, entre más electrones participen en la reacción, el valor de $\Delta E \_p$ es menor.

Otro parámetro evaluado fue la relación de las corrientes de pico anódico y catódico, i_pa/i_pc. En la Fig. 3B se presenta el análisis de pico catódico, donde la línea azul representa el valor de la corriente de pico i_pc, señalando el valor de E_pc. De forma similar, en la Fig. $3 \mathrm{C}$ se presenta el análisis de pico anódico, con el valor de la corriente de pico i_pa en color azul y en color rojo el valor de E_pa. Bajo las condiciones experimentales evaluadas dicho valor fue de 1.21 para el par redox de Pio. En el caso, que el proceso electroquímico al electrodo sea controlado por fenómenos de difusión, dicho valor es típicamente 1. Así, para los resultados obtenidos, la desviación en la relación i_pa/i_pc se debe a que el valor de i_pa es mayor al de i_pc, sugiriendo la contribución de un proceso acoplado a la oxidación de piocianina, como la adsorción de la especie sobre el electrodo. Además, en la Fig. 3C se observa incremento en el valor de i_pa con respecto a i_pc, lo que sugiere posibles complicaciones al electrodo por presencia de alguna especie de la molécula analizada. 

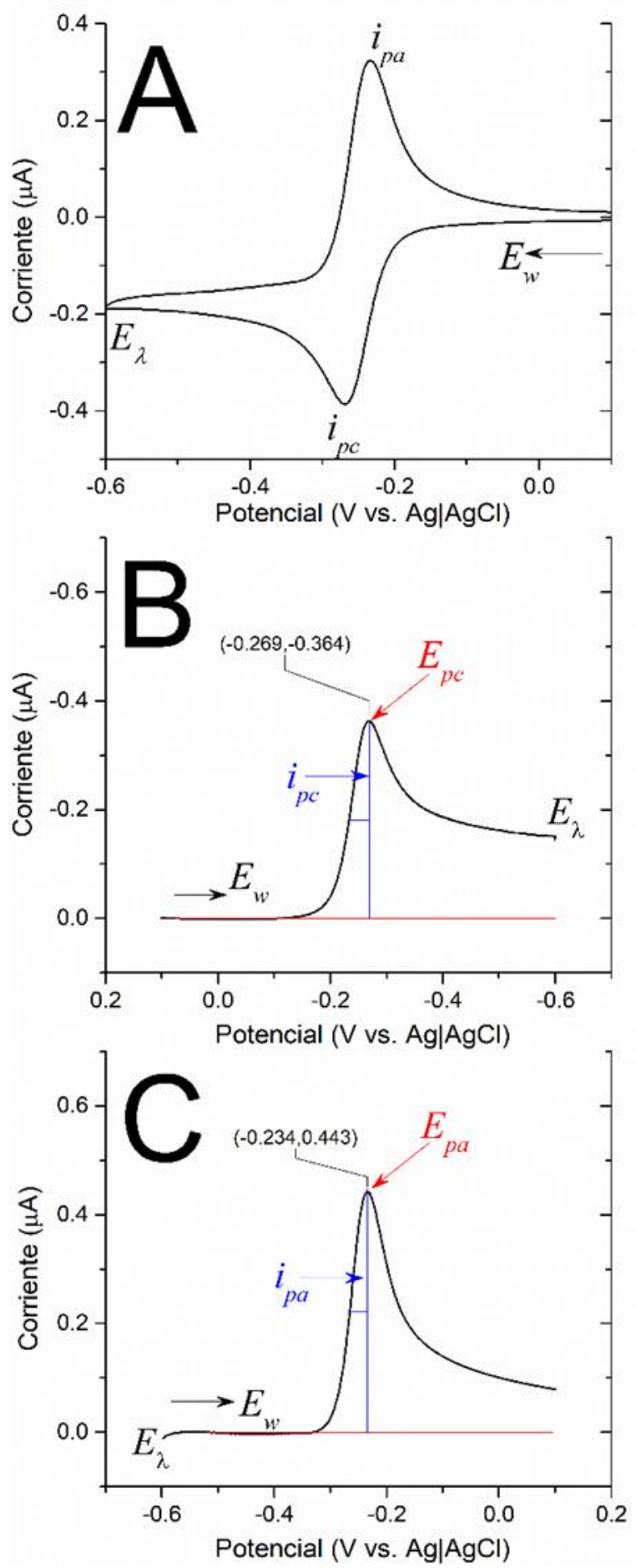

Fig. 3. (A) Voltamperometría ciclica de piocianina $(0.14 \mathrm{mM})$ en un electrodo de carbón vítreo, $\mathrm{V}=10 \mathrm{mV} / \mathrm{s}$. (B) Análisis del pico catódico substraída en 0 . (C) Análisis del pico anódico substraída en 0 .

Fig. 3. (A) Cyclic voltamperometric of pyocyanin $(0.14 \mathrm{mM})$ in glassy carbon electrode, $V=10 \mathrm{mV} / \mathrm{s}$. (B) Cathodic peak analysis subtracting in 0 . (C) Anodic peak analysis subtracting in 0 . 


\section{DISCUSIÓN}

El glicerol es una molécula pequeña y sin carga que puede transportarse a través de la membrana citoplasmática en Pseudomonas aeruginosa y de algunos microorganismos usando el transportador glicerol-3-fosfato mediante el mecanismo de transporte activo secundario (Castañeda et al., 2009; Hirakawa et al., 2018). Por ello, los microorganismos pueden utilizar al glicerol como fuente de carbono. Algunos microorganismos reportados por su capacidad para metabolizar al glicerol son Propionibacterium sp., Citrobacter sp., Clostridium sp., Enterobacter sp. o Klebsiella sp., entre otros. Esto debido a que el glicerol se metaboliza de manera oxidativa y reductiva, por enzimas como la deshidrogenasa o dehidratasa. Así, mediante la fermentación de glicerol se puede obtener una gran diversidad de compuestos como ácido propiónico, ácido succínico, butanol, propanodiol, dihidroxiacetona, entre otros (Hejna et al., 2016).

De acuerdo a los datos obtenidos en este trabajo, las tres cepas evaluadas utilizaron al glicerol crudo, incluido en el medio GC1, como única fuente de carbono para su crecimiento, sugiriendo que las impurezas presentes en el GC pueden ser toleradas $y / 0$ metabolizadas por las cepas de $P$. aeruginosa. Este efecto es contrario al efecto toxico del glicerol crudo reportado anteriormente para diversos microorganismos, donde se presenta interferencia en las vías bioquímicas relacionadas con la producción de diversos metabolitos de interés (Bharathiraja et al., 2020; Guerfali et al., 2020; Jittjang et al., 2019; Kumar et al., 2019; Iyyappan et al., 2019). Las impurezas presentes en el GC dependen del catalizador utilizado para la obtención de biodiesel, conteniendo comúnmente metanol, catalizador, materia orgánica diferente al glicerol, sales, jabones, ácido grasos y agua (Nartker et al., 2014). De las impurezas presentes en el GC1, el metanol puede inhibir el crecimiento de los microorganismos, sin embargo, la esterilización del medio de cultivo puede eliminar este compuesto (Liu et al., 2013).

En algunos trabajos se reportan valores de concentración de metanol de $0.5 \%$ $(\mathrm{p} / \mathrm{p})$ hasta más del $30 \%(\mathrm{v} / \mathrm{v})$ de metanol presente en GC (Chen et al., 2018), con un efecto negativo en la producción de biomasa. En el medio de cultivo GC se puede considerar que el contenido de metanol, que forma parte de las impurezas, fue evaporado ya que se sometió a un proceso de esterilización en autoclave (121 ${ }^{\circ} \mathrm{C}$, por 15 minutos). En experimentos realizados en este trabajo, se evaluó el efecto del metanol suplementado al medio GC1 sobre la producción de biomasa y Pio en las tres cepas de $P$. aeruginosa utilizadas, agregando una concentración del $5 \%(\mathrm{v} / \mathrm{v})$ de metanol como concentración final a los medios de cultivo GC, demostrando que hubo un efecto negativo sobre el crecimiento de NEJ01R, NEJ03R y R5, obteniéndose valores de biomasa $<0.001 \mathrm{~g} / \mathrm{L}$, para las tres cepas. Bajo tales condiciones, la producción de Pio fue nula, demostrando el efecto tóxico que tiene esa concentración de metanol sobre el crecimiento de las cepas usadas y la producción de Pio. Resultados similares son reportados para un cultivo de Ustilago maydis donde una concentración de metanol del $5 \%$ inhibió el crecimiento del microorganismo, así como la producción de glicolípidos utilizados 
como biosurfactantes en un medio de cultivo con glicerol crudo (Liu et al., 2011). Así también, se reporta que el efecto negativo del metanol sobre el crecimiento de los microorganismos es debido a que este alcohol induce una alteración en la fluidez de la membrana (Yang et al., 2014).

Otra de las impurezas presentes en el GC que pueden interferir en el crecimiento de los microorganismos son las sales como el cloruro de sodio o cloruro de potasio provenientes del catalizador. El ion sodio es necesario para el crecimiento celular ya que participa en el gradiente transmembranal de $\mathrm{pH}$, la motilidad celular y la regulación de $\mathrm{pH}$ intracelular. En un estudio realizado para evaluar el efecto de la concentración de cloruro de sodio y cloruro de potasio en la producción de dihidroxiacetona por Gluconobacter frateurii CGMCC5397, se demostró que concentraciones $>0.1 \mathrm{M}$ de cloruro de sodio afectan el crecimiento y producción de dihidroxiacetona con glicerol puro como fuente de carbono, mientras que el cloruro de potasio tiene un efecto menor. Pero, al usar GC, concentraciones de $0.11 \mathrm{M}$ de cloruro de sodio pueden tener efecto negativo para el crecimiento de Gluconobacter frateurii CGMCC5397 y producción del metabolito de interés (Liu et al., 2013). También, el GC se ha utilizado para la producción de enzimas como la lipasa B de Candida antarctica, donde se reporta que la presencia de impurezas como metanol, cloruro de sodio, mono, di y triglicéridos, así como ésteres metílicos no presentan efecto negativo en la obtención de dicha enzima (Robert et al., 2017).

De acuerdo con los datos obtenidos sobre la producción de Pio, las impurezas del GC podrían estar induciendo la biosíntesis de Pio. Entonces, sería necesario estudiar la producción de dicha molécula con glicerol puro, así como determinar la cinética de consumo de dicho glicerol por cada cepa de $P$. aeruginosa. La biotransformación del GC por microorganismos tiene una ventaja sobre su uso como precursor de síntesis química ya que los procesos de purificación del GC implican una alta inversión económica y de energía debido a los procesos de destilación y filtración por membranas para poder ser utilizado en industrias como alimentos, cosméticos y farmacéutica (Aquino et al., 2014). De acuerdo con los resultados de este trabajo y lo señalado por El-fouly et al., (2015) la síntesis biológica de Pio es una excelente alternativa para su producción industrial.

Actualmente, se considera que el uso del GC en estas industrias debe cumplir con ciertas restricciones, ya que el glicerol grado I tiene una pureza de aproximadamente $95 \%$, siendo clasificado para uso técnico como intermediario en la síntesis de químicos, mientras que el glicerol grado II debe tener porcentajes de purezas del 96 al $99.5 \%$ y es clasificado como USP (Farmacopea de Estados Unidos) para uso en la industria de alimentos, farmacéutica y cosmética y en el caso del glicerol grado III, con pureza del 99.5 al $99.8 \%$, puede ser clasificado como Kosher para la producción de alimentos y bebidas bajo (Lee et al., 2015). Por el contrario, cuando se utiliza GC para síntesis microbiana se tiene como ventaja la disminución de costos en el proceso. Además, debido a que los procesos de fermentación requieren temperaturas más bajas que otros procesos químicos, se tiene un ahorro de energía (He et al., 2017). Tal es el caso de la 
síntesis de bio-hidrógeno por Clostridium pasteurianum utilizando $10 \mathrm{~g} / \mathrm{L}$ de GC a $35^{\circ} \mathrm{C}, \mathrm{pH}=7.0$, agitación $=200 \mathrm{rpm}$, logrando producir $0.77 \pm 0.05 \mathrm{~mol} \mathrm{H} / 2 / \mathrm{mol}$ de glicerol, demostrando el alto potencial del GC para un costo efectivo de producción de bio-hidrógeno. Considerando todas estas ventajas para su producción a mayor escala usando al GC como fuente de carbono, se tendría un impacto positivo en su disponibilidad comercial a menores costos. Esto coincidiría con los resultados presentados por El-fouly et al. (2015) usando harina de semilla de algodón como medio de cultivo, que tuvo efecto positivo en los costos asociados a los procesos de extracción y purificación de la Pio producida.

Además, está demostrado que el GC es una fuente de carbono de bajo costo para la producción de biocombustibles no fósiles como bioetanol y bio-hidrógeno por Escherichia coli MG1655, alcanzando una concentración de $7.58 \pm 1.52 \mathrm{~g} / \mathrm{L}$ de etanol y $0.56 \mathrm{~mol} \mathrm{H} / \mathrm{mol}$ de glicerol, utilizando $10 \mathrm{~g} / \mathrm{L}$ de GC. En este este mismo estudio se demostró que no hubo diferencias entre el uso del GC y el glicerol grado alimenticio (Cofré et al., 2016). Otros compuestos de valor agregado que se han obtenido por biotransformación del GC son el ácido propiónico utilizando microorganismos como Propionibacterium acidipropionici, Propionibacterium acnes y Clostridium propionicum y para la síntesis de trehalosa por fermentación de Propionibacterium freudenreichii, mismo microorganismo que se puede usar para la bioproducción de ácido propiónico, ácido láctico y trehalosa (Kumar et al., 2016). En otro estudio sobre la producción de Pio se reporta que Pseudomonas aeruginosa R1 y Pseudomonas aeruginosa U3 logran producir 3.2 y $3.7 \mu \mathrm{g} / \mathrm{mL}$ de dicho metabolito, respectivamente. Estos resultados fueron obtenidos usando caldo nutritivo como medio de cultivo suplementado con glicerol puro al $3 \%$ (Elfouly et al., 2015). La producción de Pio para estas cepas es mayor que la obtenida con las tres cepas de este trabajo, ya que se utilizó un medio de cultivo general para bacterias además del glicerol puro, a diferencia del medio de cultivo GC1, que contiene únicamente glicerol crudo al $1 \%$. Destacando que el contenido de este medio de cultivo es altamente complejo, por lo que no todos los componentes pueden ser metabolizados. Para las tres cepas de $P$. aeruginosa utilizadas en este trabajo se logró la producción de Pio con el GC como única fuente de carbono, considerando a Pio un metabolito con valor agregado por sus diversas aplicaciones relacionadas a su actividad biológica como molécula redox.

Mediante técnicas cromatográficas y electroquímicas se caracterizó a la piocianina, molécula obtenida mediante biosíntesis por tres cepas silvestres de Pseudomonas aeruginosa, utilizando como única fuente de carbono un subproducto de la producción de biodiesel. Las impurezas presentes en el glicerol crudo pueden tener un efecto positivo o negativo en dicha biosíntesis dependiendo de la cepa. Las cepas NEJ01R y NEJ03R resultaron ser más eficientes en la producción de piocianina usando el medio GC1, lo cual representaría una disminución en el costo de síntesis del metabolito redox. Si se considera acoplar la bio-producción electroquímica de corriente eléctrica al proceso de producción de biodiesel a través del uso de sus residuos como el glicerol crudo, para la producción de Pio como molécula redox útil en la transferencia de electrones sobre el ánodo de un sistema bioelectroquímico, se estaría disminuyendo su 
desecho sin reuso. Esta alternativa, apoyaría la integración de una economía circular para la producción de dos energías limpias y renovables como el biodiesel y la bioelectricidad.

\section{AGRADECIMIENTOS}

Los autores agradecen al Fondo CONACYT-SEP Ciencia Básica (258159), Fondo SENER-Conacyt (246052) y Apoyo al Fortalecimiento y Desarrollo de la Infraestructura Científica (255468). FJBV y JAPG agradecen a CONACYT por las becas de posdoctorado y posgrado, respectivamente.

\section{CONFLICTO DE INTERESES}

Los autores declaran que no existe conflicto de intereses.

\section{REFERENCIAS}

Aquino E., Misturini R. \& Zachia A. 2014. Bioconversion of residual glycerol from biodiesel synthesis into 1,3-propanediol using immobilized cells of Klebsiella pneumoniae BLh-1. Renewable Energy. 72: 253-257.

Bharathirajal B., Selvakumari A.E., Jayamuthunagai J., Kumar R.P., Varjani S., Pandey A. \& Gnansounou E. 2020. Biochemical conversion of biodiesel by-product into malic acid: A waytowards sustainability. Science of the Total Environment. 709: 1-11.

Castañeda-García A, Rodríguez-Rojas A., Guelfo J. \& Jesús Blázquez J. 2009. The glycerol-3-phosphate permease GlpT is the only fosfomycin transporter in Pseudomonas aeruginosa. Journal of Bacteriology. 191: 6968 - 6974.

Cofré O., Ramírez M., Gomez J.M. \& Cantero D. 2016. Pilot scale fed-batch fermentation in a closed loop mixed reactor for the biotransformation of crude glycerol into ethanol and hydrogen by Escherichia coli MG1655. Biomass and Bioenergy. 91: 37-47.

Chen J., Zhang X., Tyagi R.D. \& Drogui P. 2018. Utilization of methanol in crude glycerol to assist lipid production in non-sterilized fermentation from Trichosporon oleaginosus. Bioresource Technology. 253: 8-15.

Chozhavendhan S., Praveen Kumar R., Elavazhagan S., Barathiraja B., Jayakumar M. \& Varjani S.J. 2018. Utilization of crude glycerol from biodiesel industry for the production of value-added bioproducts. In: Waste to wealth, energy, environment and sustainability. (Editores, Singhania R., Agarwal R., Kumar R., Sukumaran R.) Springer, Singapore. doi.org/10.1007/978-981-10-74318 8. 
El-Fouly M.Z., Sharaf A.M., Shahin A.A., El-Bialy H.A. \& Omara A.M. 2015. Biosynthesis of pyocyanin pigment by Pseudomonas aeruginosa. Journal of Radiation Research and Applied Sciences. 8: 36-48.

Guerfali M., Ines Ayadi I., Sassi H.E., Belhassen A., Gargouri A. \& Belghith H. 2020. Biodiesel-derived crude glycerol as alternative feedstock for single cell oil production by the oleaginous yeast Candida viswanathii Y-E4. Industrial Crops \& Products. 145: 1-10.

He Q., McNutt J. \& Yang J. 2017. Utilization of the residual glycerol from biodiesel production for renewable energy generation. Renewable and Sustainable Energy Reviews. 71: 63-76.

Hejna A., Kosmela P., Formela K. \& Piszczyk L. 2016. Potential applications of crude glycerol in polymer technology - Current state and perspectives. Renewable and Sustainable Energy Reviews. 66: 449-475.

Hirakawa H., Kurabayashi K., Koichi Tanimoto K., \& Tomita H. 2018. Oxygen limitation enhances the antimicrobial activity of fosfomycin in Pseudomonas aeruginosa following overexpression of glpT which encodes glycerol-3phosphate/fosfomycin symporter. Frontiers in Microbiology. 9: 1-10.

Jittjang S., Jiratthiticheep I., Kajonpradabkul P., Tiatongjitman T., Siriwatwechakul W. \& Boonyarattanakalin S. 2019. Effect of $\mathrm{NaCl}$ removal from biodiesel-derived crude glycerol by ion exchange to enhance dihydroxyacetone production by Gluconobacter thailandicus in minimal medium. Journal of Chemical Technology and Biotechnology. 95: 281-288.

Kurachi M. 1958. Studies on the biosynthesis of pyocyanine. II. Isolation and determination of pyocyanine. Bulletin of the Institute for Chemical Research, Kyoto University 36: 174-187.

Kondaveeti S., Kim I.W., Otari S., Patel S.K.S., Pagolu R., Losetty V., Kalia V.C. \& Lee J.K. 2019. Co-generation of hydrogen and electricity from biodiesel process effluents. International Journal of Hydrogen Energy. 44: 27285-27296.

Kumar G., Shankar U. \& Budhiraja A. 2016. Bioconversion technologies of crude glycerol to value added industrial products. Biotechnology Reports 9: 9-14.

Kumar L., Kumar Y., Tyagi R.D. \& Zhang X. 2019. A review on variation in crude glycerol composition, bio- valorization of crude and purified glycerol as carbon source for lipid production. Bioresource Technology. 293: 1- 11.

lyyappan J., Bharathiraja B., Baskar G. \& Kamalanaban E. 2019. Process optimization and kinetic analysis of malic acid production from crude glycerol using Aspergillus niger. Bioresource Technology. 281: 18-25. 
Lee C.S., Aroua M.K., Daud W.M.A.W., Cognet P. \& Pérès P. 2015. A review: Conversion of bioglycerol into 1,3-propanediol via biological and chemical method. Renewable and Sustainable Energy Reviews. 42: 63-972.

Liu Y.P., Sun Y., Tan C., Li H., Zheng X.J., Jin K.Q. \& Wang G. 2013. Efficient production of dihydroxyacetone from biodiesel-derived crude glycerol by newly isolated Gluconobacter frateurii. Bioresource Technology. 142: 384-389.

Liu Y., Koh C. \& Ji L. 2011. Bioconversion of crude glycerol to glycolipids in Ustilago maydis. Bioresource Technology. 102: 3927-3933.

Mangayil R., Efimova E., Konttinen J. \& Santala V. 2019. Co-production of 1, 3 propanediol and long-chain alkyl esters from crude glycerol. New Biotechnology. 53: 81-89.

Nartker S., Ammerman M., Aurandt J., Stogsdil M., Hayden O. \& Antle C. 2014. Increasing biogas production from sewage sludge anaerobic co-digestion process by adding crude glycerol from biodiesel industry. Waste Management. 34: 25672571.

Oziat J., Gougis M., Malliaras G.G. \& Mailley P. 2017. Electrochemical characterizations of four main redox-metabolites of Pseudomonas aeruginosa. Electroanalysis. 29: 1332-1340.

Patil S., Nikama M., Patil H., Anokhina T., Kochetkov V. \& Chaudharia A. 2017. Bioactive pigment production by Pseudomonas spp. MCC 3145: Statistical media optimization, biochemical characterization, fungicidal and DNA intercalation-based cytostatic activity. Process Biochemistry. 58: 298-305.

Robert J.M., Senna L., Machado A., Machado C., Volcan A., Araripe G., Francisco Valero F. \& Guimarães F. 2017. Production of recombinant lipase B from Candida antarctica in Pichia pastoris under control of the promoter PGK using crude glycerol from biodiesel production as carbon source. Biochemical Engineering Journal. 118: 123-131.

Sarma S., Ortega D., Minton N.P., Dubey V.K. \& Moholkar V.S. 2019. Homologous overexpression of hydrogenase and glycerol dehydrogenase in Clostridium pasteurianum to enhance hydrogen production from crude glycerol. Bioresource Technology. 284:168-177.

Shen H., Yong X., Chen Y., Liao Z., Si R., Zhou J., Wang S., Yong Y., OuYang P. \& Zheng T. 2014. Enhanced bioelectricity generation by improving pyocyanin production and membrane permeability through sophorolipid addition in Pseudomonas aeruginosa-inoculated microbial fuel cells. Bioresource Technology. 167: 490-494. 
Uprety B.K., Venkatesagowda B. \& Rakshit S.K. 2017. Current prospects on production of microbial lipid and other value-added products using crude glycerol obtained from biodiesel industries. BioEnergy Research. 10: 1117-1137.

Webster T.A. \& Goluch E.D. 2012. Electrochemical detection of pyocyanin in nanochannels with integrated palladium hydride reference electrodes. Lab chip. 12:5195-5201.

Xu L.Q., Zeng J.W., Jiang C.H., Wang H., Li Y.Z., Wen W.H., Li J.H., Wang F., Ting W.J., Sun Z.Y. \& Huang C.Y. 2017. Isolation and determination of four potential antimicrobial components from Pseudomonas aeruginosa extracts. International Journal of Medical Sciences. 14: 1368-1374.

Yang X., Jin G., Gong Z., Shen H., Bai F. \& Zhao Z.K. 2014. Recycling biodieselderived glycerol by the oleaginous yeast Rhodosporidium toruloides $Y 4$ through the two-stage lipid production process. Biochemical Engineering Journal. 91, 15: 8691.

Yong X.Y., Yan Z.Y., Shen H.B., Zhou J., Wua X.Y., Zhang L.J., Zheng T., Jiang M., Wei P., Jia H.H. \& Yong Y.C. 2017. An integrated aerobic-anaerobic strategy for performance enhancement of Pseudomonas aeruginosa-inoculated microbial fuel cell. Bioresource Technology. 241: 1191-1196. 